\title{
Original Reserach Article
}

\section{Synthesis, characterization and antimicrobial activity of novel tetrazoles clubbed with pyrimidine}

\author{
N.D. Bhoge ${ }^{1, *}$, B.K. Magare ${ }^{2}$, P. B. Mohite ${ }^{3}$ \\ ${ }^{1}$ Dept. of Chemistry, Shri Dnyaneshwar Mahavidyalaya, Newasa, Maharashtra, India \\ ${ }^{2}$ Dept. of Chemistry, Shivaji Arts, Commerce and Science Collège, Kannad, Maharashtra, India \\ ${ }^{3}$ Dept. of Pharmaceutical Chemistry, Mula Education Societys College of Pharmacy, Ahemadnagar, Maharashtra, India
}

\section{A R T I C L E I N F O}

Article history:

Received 11-07-2021

Accepted 07-09-2021

Available online 19-11-2021

\section{Keywords:}

Antimicrobial Activity

Pyrimidine

Tetrazole etc

\begin{abstract}
A B S T R A C T
An attempt was made to synthesize pyrimidine tetrazole derivatives of pharmaceutical interest by oxidative cyclization of chalcones with adequate yield and purity, prompted by the diversity of their wider usage and the fact that they are an integral part of genetic content. The present work involves the reaction of 5-(2,6dimethylphenyl)-1H-tetrazole with acetic anhydride to yield 1-[5-(2,6-dimethylphenyl)-1H-tetrazol-1-yl] ethanone (1) and which then treated with different aromatic aldehydes in presence of alkaline medium to chalcones (2a-f). Reaction of chalcones (2a-f) with urea and thiourea to produce 5-[5-(2,6-dimethylphenyl)1H-tetrazol-1-yl]-4-(substituted aryl ) pyrimidin-2-ol (3a-f) and 5-[5-(2,6-dimethylphenyl)-1H-tetrazol-1yl]-4-(substituted aryl) pyrimidin-2-thiol (4a-f) respectively. All compounds were characterized by infrared spectroscopy (IR), ${ }^{1} \mathrm{H}$ nuclear magnetic resonance (NMR), and mass spectrometry (MS) to prove the structure and assessed in vitro for their efficacy as antibacterial and antifungal activity against four bacteria. The compounds $3 \mathrm{c}, 3 \mathrm{~d}$ and $3 \mathrm{f}$ and compounds $4 \mathrm{c}, 4 \mathrm{~d}$ and $4 \mathrm{f}$ possess very good activity against $S$. aureus and E. coli and the compounds $3 \mathrm{e}, 3 \mathrm{c}$ and $3 \mathrm{a}$ and compounds $4 \mathrm{e}, 4 \mathrm{~b}$ and $4 \mathrm{c}$ possess very good activity against fungi Candida albicans and Aspergillus niger.
\end{abstract}

This is an Open Access (OA) journal, and articles are distributed under the terms of the Creative Commons Attribution-NonCommercial-ShareAlike 4.0 License, which allows others to remix, tweak, and build upon the work non-commercially, as long as appropriate credit is given and the new creations are licensed under the identical terms.

For reprints contact: reprint@ipinnovative.com

\section{Introduction}

Since they are a diverse group of natural and synthetic products, many of which have biological applications, nitrogen-containing heterocycles are extremely important. Pyrimidine, which is present in DNA and RNA, has a variety of pharmacological properties, including bactericide, fungicide, vermicide, insecticide, anticancer, and antiviral. ${ }^{1}$ Some pyrimidine derivatives reported as Anti-HIV-1 agents, ${ }^{2}$ antileishmanial, ${ }^{3}$ Anti-inflammatory, ${ }^{4}$ Anticancer, ${ }^{5}$ antimicrobial Antimalarial. ${ }^{6}$ To date, a wide range of pyrimidine and pyrimidine-fused heterocyclic compounds have been documented to have anticancer

\footnotetext{
* Corresponding author.

E-mail address: nitinbhoge4550@gmail.com (N. D. Bhoge).
}

activity through a variety of mechanisms and targets. ${ }^{7-9}$ Pyrimidine derivatives ${ }^{10,11}$ have played a significant role in the evolution of heterocyclic chemistry and have been widely used as pharmacophores and synthons in organic chemistry. A considerable amount of research effort has been centered on these nuclei due to their flexible chemotherapeutic significance. There has been significant progress in this area since the discovery of many synthetic and semi-synthetic antibacterial sulfa products, nitrofuranes, penicillins, cephalosporins, tetracyclines, macrolides, oxazolidinones, and antifungal agents such as fluconazole, ketoconazole, and miconazole, as well as amphotericin B. Despite advancements in antibacterial and antifungal therapies, most antimicrobial drugs still have a long way to go. Antibiotic overuse has 
resulted in the emergence of multidrug-resistant microbial pathogens. ${ }^{12}$ Pyrimidine based heterocycles are potential bioactive molecules and exhibit antimicrobial, ${ }^{13,14}$ anti-inflammatory, ${ }^{11}$ antioxidant, ${ }^{15}$ anticancer, ${ }^{16}$ antihypertensive $^{17}$ and anticonvulsant. ${ }^{18}$ Tetrazole has a great importance as it is bioactive molecules and exhibit antimicrobial, ${ }^{19}$ anti-bacterial ${ }^{20}$ anti-inflammatory ${ }^{21}$, antioxidant, ${ }^{22}$ anticancer ${ }^{23}$ ant tubercular, ${ }^{24}$ anti-fungal, ${ }^{24}$ antihypertensive $^{25}$, anticonvulsant ${ }^{26}$ and also act as enzyme inhibitors. Inspired from these facts, in present work an attempt is being made to synthesize pyrimidine's containing tetrazole and evaluate for antimicrobial activity which has not been reported yet. Hence the present work deals with the reaction of 1-[5-(2,6-dimethylphenyl)1H-tetrazol-1-yl] ethanone (1) with different aromatic aldehydes in presence of alkaline medium to form (2E)-1[5-(2,6-dimethylphenyl)- 1H-tetrazol-1-yl]-3-(substituted aryl)prop-2-en-1-one (2a-f). Reaction of (2a-f) with urea and thiourea to produce 5-[5-(2,6-dimethylphenyl)- $1 \mathrm{H}$ tetrazol-1-yl]-4-(substituted aryl )pyrimidin-2-ol (3a-f) and 5-[5-(2,6-dimethylphenyl)-1H-tetrazol-1-yl]- 4-(substituted aryl) pyrimidin-2-thiol (4a-f) respectively. The structure of all the various synthesized compounds were assigned on the basis of IR, ${ }^{1} \mathrm{H}$ NMR spectral data and elemental analysis. These compounds were screened for their antimicrobial activity.

\section{Materials and Methods}

Melting points were determined with open capillary. FT-IR spectra were recorded on a Jasco model 4010 spectrophotometer, ${ }^{1} \mathrm{H}$ NMR spectra were recorded in DMSO on a Varian mercury FT-NMR model YH- 300 instrument using TMS as internal standard. Mass spectra were recorded on GC-MS auto tune EI instrument.

\subsection{Synthetic procedur}

\subsubsection{General procedure for the preparation}

(2E)-1-[5-(2,6-dimethylphenyl)-

1H-tetrazol-1-yl]-3-(substituted aryl) prop-2-en-1-one derivatives [2a-f]

A solution of 5-(2,6-dimethylphenyl)-1H-tetrazole $(8.5 \mathrm{~g}, 0.05$ moles $)$ and heterocyclic aldehydes $(0.05$ mole) in ethanol $(12 \mathrm{ml})$ was cooled to 5 to $10^{\circ} \mathrm{C}$ in an ice bath. The cooled solution was treated with drop wise addition of aqueous sodium hydroxide ( $5 \mathrm{ml}, 50 \%$ ). The reaction mixture was magnetically stirred for $30 \mathrm{~min}$ and then left over night. The resulting dark solution was diluted with ice water and carefully acidified using diluted hydrochloric acid. The tetrazole analogues of chalcone which crystallized were collected by filtration after washing with sodium bicarbonate and water. It was further purified by crystallization from ethanol.

\subsubsection{Synthesis of}

5-[5-(2,6-dimethylphenyl)-1H-tetrazol-1-yl]-4- (substituted ary) pyrimidin-2-ol derivatives [3a-f]

To a solution of 2E)-1-[5-(2,6-dimethylphenyl)-1H-tetrazol1-yl]- 3-(substituted aryl)prop-2-en-1-one derivatives (2af), $(0.01$ mole $)$ in anhydrous ethanol $(50 \mathrm{~mL})$, urea $(0.01$ mole)and aqueous sodium hydroxide (0.01 mole). The reaction mixture was refluxed for $5 \mathrm{hrs}$ and poured into ice cold water the product obtained was filtered, washed with water and crystallized from aqueous ethanol. The purity of the compound was established by TLC using a mixture of hexane and ethyl acetate (7:3) as a mobile phase.

\subsubsection{Synthesis of}

5-[5-(2,6-dimethylphenyl)-1H-tetrazol-1-yl]-4- (substituted aryl) pyrimidin-2-thiol derivatives [4a-f]

To a solution of 2E)-1-[5-(2,6-dimethylphenyl)-1H-tetrazol1-yl]-3- (substituted aryl) prop-2-en-1-one derivatives (2a$\mathrm{f}),(0.01 \mathrm{~mole})$ in anhydrous ethanol $(50 \mathrm{~mL})$, thiourea $(0.01$ mole) and aqueous sodium hydroxide (0.01 mole). The reaction mixture was refluxed for $5 \mathrm{hrs}$ and poured into ice cold water the product obtained was filtered, washed with water and crystallized from aqueous ethanol. The purity of the compound was established by TLC using a mixture of hexane and ethyl acetate (6:4) as a mobile phase.

\subsection{Antibacterial and antifungal activity}

All the newly synthesized compounds were screened for antimicrobial activity against both gram positive $\mathrm{S}$. aureus and gram negative E.coli bacteria and antifungal activity against $C$. albicans and $A$. niger according to cup plate $\operatorname{method}^{13}$ at a concentration $100 \mathrm{ug} / 0.1 \mathrm{ml}$ respectively. Streptomycin and clotrimazole were used as standard for comparison of antibacterial and antifungal activity ${ }^{14}$ Indian Pharmacopoeia $^{15}$. Solvent dimethyl sulphoxide (DMSO) was used as control. The results of screening are given inTable 2.

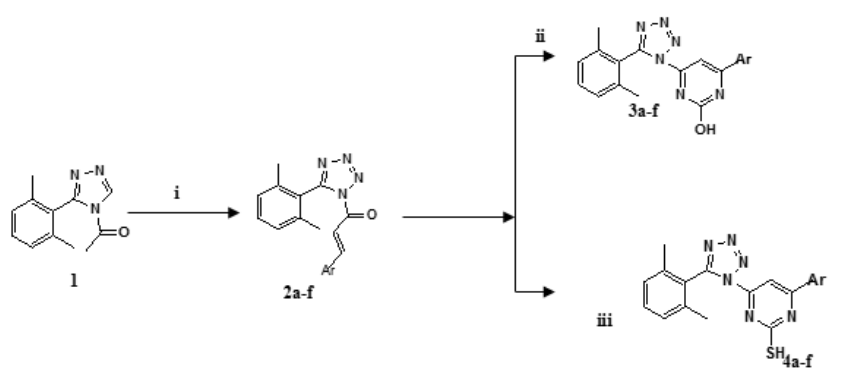

\subsection{Synthesis and spectral characterization}

A series of 5-[5-(2,6-dimethylphenyl)-1H-tetrazol-1-yl]4-(substituted aryl) pyrimidin-2-ol (3a-f) and 5-[5-(2,6dimethylphenyl)-1H-tetrazol-1-yl]-4-(substituted aryl) 
Table 1: eagents and conditions:i) Ar-CHO/ EtOH, ii) Urea/Aq. NaOH, iii) Thiourea/Aq. NaOH

\begin{tabular}{|c|c|c|c|c|c|c|c|c|c|}
\hline \multirow{2}{*}{ Comp no } & \multirow{2}{*}{$\mathbf{R}$} & \multirow{2}{*}{$\begin{array}{c}\text { Mole. } \\
\text { Formula }\end{array}$} & \multirow{2}{*}{ MW } & \multirow{2}{*}{$\%$ Yield } & \multirow{2}{*}{ M.P. ${ }^{0} \mathrm{C}$} & \multirow{2}{*}{$\mathbf{R}_{\mathbf{f}}$} & \multicolumn{3}{|c|}{ Found (Calcd) \% } \\
\hline & & & & & & & $\mathrm{C}$ & $\mathrm{H}$ & $\mathrm{N}$ \\
\hline $3 a$ & Fig1 & $\mathrm{C}_{17} \mathrm{H}_{15} \mathrm{~N}_{7} 0$ & 333 & 72 & 164 & 0.64 & $\begin{array}{c}61.20 \\
(61.25)\end{array}$ & $\begin{array}{c}4.50 \\
(4.54)\end{array}$ & $\begin{array}{c}29.39 \\
(29.41)\end{array}$ \\
\hline $3 b$ & Fig2 & $\mathrm{C}_{17} \mathrm{H}_{14} \mathrm{~N}_{6} \mathrm{O}_{2}$ & 334 & 62 & 172 & 0.65 & $\begin{array}{c}61.04 \\
(61.07)\end{array}$ & $\begin{array}{c}4.20 \\
(4.22)\end{array}$ & $\begin{array}{c}25.12 \\
(25.14)\end{array}$ \\
\hline $3 \mathrm{c}$ & Fig3 & $\mathrm{C}_{18} \mathrm{H}_{16} \mathrm{~N}_{6} \mathrm{O}_{2}$ & 348 & 65 & 174 & 0.73 & $\begin{array}{c}62.01 \\
(62.06)\end{array}$ & $\begin{array}{c}4.60 \\
(4.63)\end{array}$ & $\begin{array}{c}24.08 \\
(24.12)\end{array}$ \\
\hline $3 d$ & Fig4 & $\mathrm{C}_{18} \mathrm{H}_{16} \mathrm{~N}_{6} 0 \mathrm{~S}$ & 364 & 60 & 166 & 0.68 & $\begin{array}{c}59.29 \\
(59.32)\end{array}$ & $\begin{array}{c}4.38 \\
(4.43)\end{array}$ & $\begin{array}{c}23.04 \\
(23.06)\end{array}$ \\
\hline $3 e$ & Fig5 & $\mathrm{C}_{18} \mathrm{H}_{16} \mathrm{~N}_{6} 0 \mathrm{~S}$ & 364 & 64 & 164 & 0.65 & $\begin{array}{c}59.29 \\
(59.32)\end{array}$ & $\begin{array}{c}4.38 \\
(4.43)\end{array}$ & $\begin{array}{c}23.04 \\
(23.06)\end{array}$ \\
\hline $3 \mathrm{f}$ & Fig6 & $\mathrm{C}_{18} \mathrm{H}_{15} \mathrm{~N}_{7} 0$ & 345 & 74 & 150 & 0.75 & $\begin{array}{c}62.57 \\
(62.60)\end{array}$ & $\begin{array}{c}4.33 \\
(4.38)\end{array}$ & $\begin{array}{c}28.36 \\
(28.39)\end{array}$ \\
\hline $4 a$ & Fig7 & $\mathrm{C}_{17} \mathrm{H}_{14} \mathrm{~N}_{7} \mathrm{~S}$ & 348 & 72 & 166 & 0.62 & $\begin{array}{c}58.41 \\
(58.44)\end{array}$ & $\begin{array}{c}4.29 \\
(4.33)\end{array}$ & $\begin{array}{c}28.03 \\
(28.06)\end{array}$ \\
\hline $4 b$ & Fig8 & $\mathrm{C}_{17} \mathrm{H}_{16} \mathrm{~N}_{6} \mathrm{OS}$ & 350 & 66 & 180 & 0.66 & $\begin{array}{c}58.22 \\
(58.27)\end{array}$ & $\begin{array}{c}4.00 \\
(4.03)\end{array}$ & $\begin{array}{c}23.95 \\
(23.98)\end{array}$ \\
\hline $4 c$ & Fig9 & $\mathrm{C}_{18} \mathrm{H}_{16} \mathrm{~N}_{6} 0 \mathrm{~S}$ & 364 & 68 & 178 & 0.72 & $\begin{array}{c}59.26 \\
(59.32)\end{array}$ & $\begin{array}{c}4.41 \\
(4.43)\end{array}$ & $\begin{array}{c}23.05 \\
(23.06)\end{array}$ \\
\hline $4 d$ & Fig10 & $\mathrm{C}_{18} \mathrm{H}_{16} \mathrm{~N}_{6} \mathrm{~S}_{2}$ & 380 & 65 & 166 & 0.68 & $\begin{array}{c}56.80 \\
(56.82)\end{array}$ & $\begin{array}{c}4.20 \\
(4.24)\end{array}$ & $\begin{array}{c}22.06 \\
(22.09)\end{array}$ \\
\hline $4 \mathrm{e}$ & Fig11 & $\mathrm{C}_{18} \mathrm{H}_{16} \mathrm{~N}_{6} \mathrm{~S}_{2}$ & 380 & 64 & 168 & 0.58 & $\begin{array}{c}56.76 \\
(56.82)\end{array}$ & $\begin{array}{c}4.22 \\
(4.24)\end{array}$ & $\begin{array}{c}25.04 \\
(22.09)\end{array}$ \\
\hline $4 \mathrm{f}$ & Fig12 & $\mathrm{C}_{18} \mathrm{H}_{15} \mathrm{~N}_{7} \mathrm{~S}$ & 361 & 72 & 185 & 0.75 & $\begin{array}{c}59.77 \\
(59.82)\end{array}$ & $\begin{array}{c}4.14 \\
(4.18)\end{array}$ & $\begin{array}{c}27.10 \\
(27.13)\end{array}$ \\
\hline
\end{tabular}

Table 2: Antibacterial and antifungal data of pyrimidine

\begin{tabular}{|c|c|c|c|c|}
\hline \multirow[t]{2}{*}{ Comp. } & \multicolumn{3}{|c|}{ Zone of inhibition in $\mathbf{m m}$ at $100 \mu \mathrm{g} / \mathbf{0 . 1 m l}$} & \multirow[b]{2}{*}{ A. niger } \\
\hline & S. aureus & E. coli & C. albicans & \\
\hline $3 \mathrm{a}$ & 14 & 12 & 20 & 18 \\
\hline $3 b$ & 15 & 13 & 20 & 16 \\
\hline $3 \mathrm{c}$ & 18 & 16 & 21 & 15 \\
\hline $3 d$ & 16 & 14 & 18 & 13 \\
\hline $3 e$ & 18 & 15 & 21 & 22 \\
\hline $3 \mathrm{f}$ & 20 & 18 & 16 & 12 \\
\hline $4 a$ & 16 & 13 & 15 & 13 \\
\hline $4 b$ & 16 & 14 & 20 & 16 \\
\hline $4 c$ & 18 & 17 & 20 & 14 \\
\hline $4 d$ & 16 & 15 & 18 & 12 \\
\hline
\end{tabular}

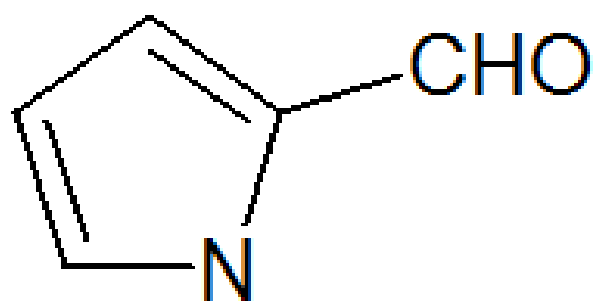

$\mathrm{H}$

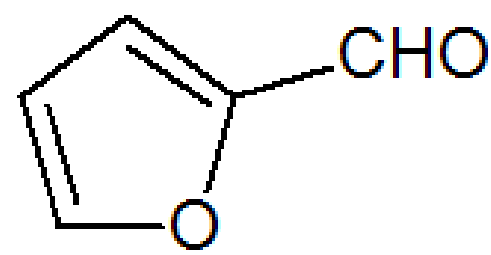

Fig. 2: Table1+R+Show $3 b$

Fig. 1: Table1+R+Show 3a 

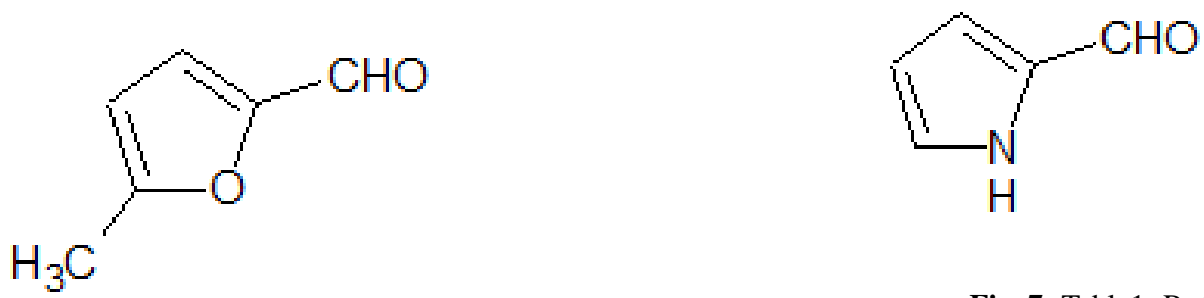

Fig. 7: Table1+R+Show 4a

Fig. 3: Table1+R+Show 3c
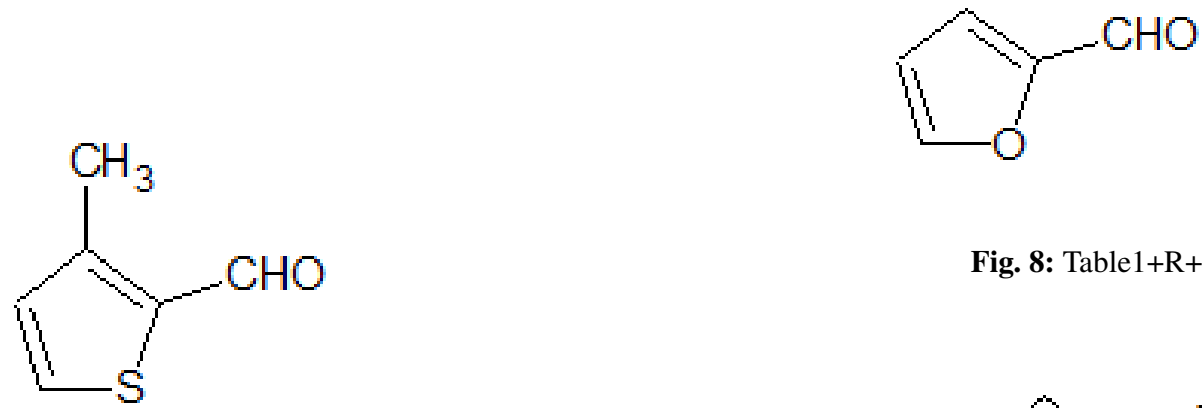

Fig. 8: Table1+R+Show $4 \mathrm{~b}$

Fig. 4: Table1+R+Show 3d
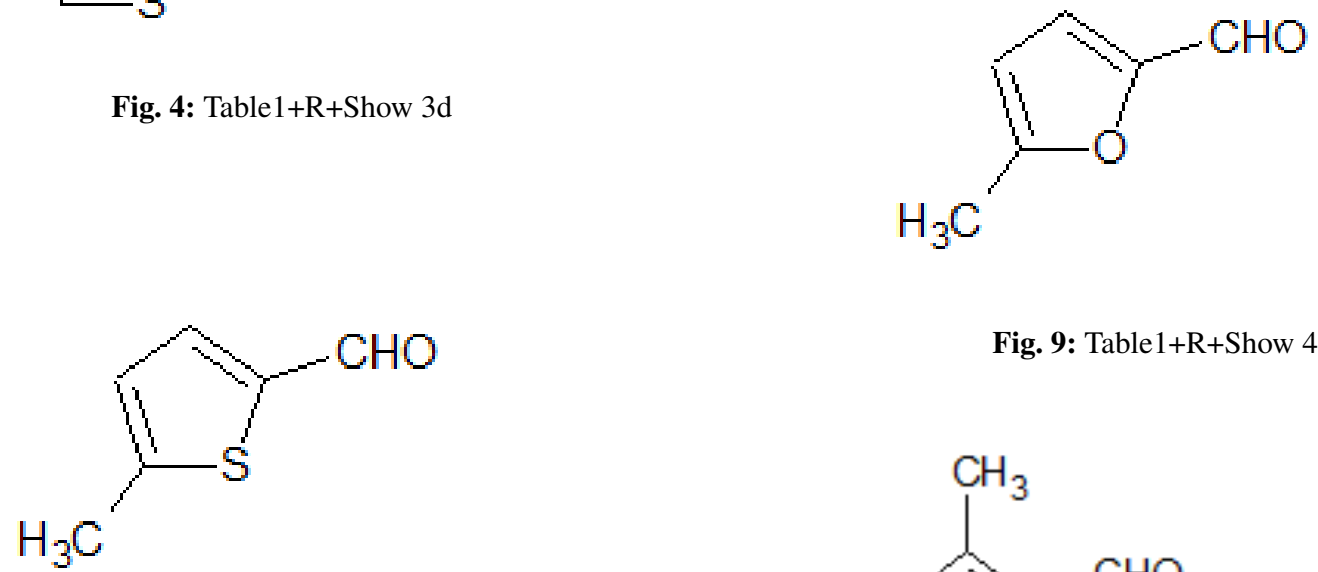

Fig. 9: Table1+R+Show 4c

Fig. 5: Table1+R+Show 3e<smiles>Cc1ccsc1C=O</smiles>

Fig. 10: Table1+R+Show $4 d$<smiles>O=Cc1ccccn1</smiles><smiles>Cc1ccc(C=O)s1</smiles>

Fig. 6: Table1+R+Show $3 f$

Fig. 11: Table1+R+Show 4e 
<smiles>O=Cc1ccccn1</smiles>

Fig. 12: Table1+R+Show $4 \mathrm{f}$

pyrimidin-2-thiol (4a-f) were synthesized from chalcones of 5-(2,6-dimethylphenyl)-1H-tetrazole. All synthesis steps are presented in scheme 1 . The IR spectra shows 1542 $(\mathrm{C}=\mathrm{N}), 1445(\mathrm{C}=\mathrm{C})$ providing the strong evidence for pyrimidine ring. ${ }^{1} \mathrm{H}$ NMR spectrum shows $7.10-7.58 \mathrm{ppm}$ for aromatic protons and $9.7 \mathrm{ppm}$ for $\mathrm{OH}$ protons and 13.4 for $\mathrm{SH}$ protons were observed at expected signals.

\subsubsection{3a:5-[5-(2,6-dimethylphenyl)-1H-tetrazol-1-yl]-4- (1H-pyrrol-2-yl)pyrimidin-2-ol}

IR: $3745(\mathrm{OH}), 3050(\mathrm{Ar}-\mathrm{CH}), 1540(\mathrm{C}=\mathrm{N}), 1435(\mathrm{C}=\mathrm{C})$, 1286(N-N=N-),1120 and 1145 (Tetrazole ring), ${ }^{1} \mathrm{H}$ NMR: $2.35\left(\mathrm{~d}, 6 \mathrm{H}, \mathrm{CH}_{3}\right), 6.5-6.8(\mathrm{~d}, 1 \mathrm{H}, \mathrm{CH}=\mathrm{CH}) \quad 7.30 \quad-8.40$ $(\mathrm{m}, 8 \mathrm{H}$, Ar-H), $9.5(1 \mathrm{H}$, Ar- $\mathrm{OH})$, Mass spectrum $(\mathrm{m} / \mathrm{z})$ molecular ion peak at 332 and isotopic peak at 333 .

\subsubsection{3b: 5-[5-(2,6-dimethylphenyl)-1H-tetrazol-1-yl]-4-} (furan-2-yl)pyrimidin-2-ol:

IR: $3744(\mathrm{OH}), 3054$ (Ar-CH), $1538(\mathrm{C}=\mathrm{N}), 1436(\mathrm{C}=\mathrm{C})$, $1280(\mathrm{~N}-\mathrm{N}=\mathrm{N}-), 1120$ and 1145 (Tetrazole ring) , 780(C$\mathrm{Cl}) .,{ }^{1} \mathrm{H}$ NMR: $2.35\left(\mathrm{~d}, 6 \mathrm{H}, \mathrm{CH}_{3}\right), 6.5-6.8(\mathrm{~d}, 1 \mathrm{H}, \mathrm{CH}=\mathrm{CH})$ $7.30-8.40$ (m ,7H, Ar-H), 9.5 (1H, Ar- $\mathrm{OH})$, Mass spectrum $(\mathrm{m} / \mathrm{z})$ molecular ion peak at 334 and and isotopic peak at 315 .

\subsubsection{3c: 5-[5-(2,6-dimethylphenyl)-1H-tetrazol-1-yl]-4-} (5-methylfuran-2-yl)pyrimidin-2-ol:

IR: $3742(\mathrm{OH}), 3056(\mathrm{Ar}-\mathrm{CH}), 1536(\mathrm{C}=\mathrm{N}), 1432(\mathrm{C}=\mathrm{C})$, 1282(N-N=N-),1245(-OCH3) ,1120 and 1145(Tetrazole ring) ${ }^{1} \mathrm{H}$ NMR: 2.35 (d, 9H, $\left.\mathrm{CH}_{3}\right), 6.5-6.8(\mathrm{~d}, 1 \mathrm{H}, \mathrm{CH}=\mathrm{CH})$ $7.30-8.40$ (m ,7H, Ar-H), 9.5 (1H, Ar- $\mathrm{OH})$,, Mass spectrum $(\mathrm{m} / \mathrm{z})$ molecular ion peak at 348 .

\subsubsection{3d: 5-[5-(2,6-dimethylphenyl)-1H-tetrazol-1-yl]-4-} (5-methylthiophen-2-yl)pyrimidin-2-ol

IR: $3740(\mathrm{OH}), 3058(\mathrm{Ar}-\mathrm{CH}), 1546(-\mathrm{NO} 2), 1530(\mathrm{C}=\mathrm{N})$, $1441(\mathrm{C}=\mathrm{C}), 1278(\mathrm{~N}-\mathrm{N}=\mathrm{N}-), 1120$ and 1145 (Tetrazole ring) , ${ }^{1} \mathrm{H}$ NMR: 2.35 (d, 9H, $\left.\mathrm{CH}_{3}\right), 6.5-6.8(\mathrm{~d}, 1 \mathrm{H}, \mathrm{CH}=\mathrm{CH}) 7.30$ $8.40(\mathrm{~m}, 7 \mathrm{H}$, Ar-H), $9.5(1 \mathrm{H}$, Ar- $\mathrm{OH})$, , Mass spectrum $(\mathrm{m} / \mathrm{z})$ molecular ion peak at 364 .

\subsubsection{3e:5-[5-(2,6-dimethylphenyl)-1H-tetrazol-1-yl]-4- (3-methylthiophen-2-yl)pyrimidin-2-ol}

IR: $3735(\mathrm{OH}), 3054(\mathrm{Ar}-\mathrm{CH}), 1544(\mathrm{C}=\mathrm{N}), 1435(\mathrm{C}=\mathrm{C})$, 1331(-N(CH3)2,1284 (N-N=N-),1120 and 1145(Tetrazole ring) ${ }_{1}^{1} \mathrm{H}$ NMR: 2.35 (d, 9H, $\left.\mathrm{CH}_{3}\right), 6.5-6.8(\mathrm{~d}, 1 \mathrm{H}, \mathrm{CH}=\mathrm{CH})$ $7.30-8.40$ (m,7H, Ar-H), $9.5(1 \mathrm{H}$, Ar- $\mathrm{OH})$, Mass spectrum $(\mathrm{m} / \mathrm{z})$ molecular ion peak at 364 .

2.3.6. 3f: 5-[5-(2,6-dimethylphenyl)-1H-tetrazol-1-yl]-4(pyridin-2-yl)pyrimidin-2-ol

IR: $3741(\mathrm{OH}), 3052$ (Ar-CH), $1544(\mathrm{C}=\mathrm{N}), 1436(\mathrm{C}=\mathrm{C})$, 1355(CH3), 1284 (N-N=N-),1120 and 1145(Tetrazole ring), ${ }^{1} \mathrm{H}$ NMR: $2.35\left(\mathrm{~d}, 6 \mathrm{H}, \mathrm{CH}_{3}\right), 6.5-6.8(\mathrm{~d}, 1 \mathrm{H}, \mathrm{CH}=\mathrm{CH})$ $7.30-8.40$ (m ,9H, Ar-H), 9.5 (1H, Ar- $\mathrm{OH})$, Mass spectrum $(\mathrm{m} / \mathrm{z})$ molecular ion peak at 345 .

\subsubsection{4a:5-[5-(2,6-dimethylphenyl)-1H-tetrazol-1-yl]-4-} (1H-pyrrol-2-yl)pyrimidin-2-thiol

IR:3055 (Ar-CH), 1538(C=N), 1436(C=C), $1288(\mathrm{~N}-\mathrm{N}=\mathrm{N}-$ ), 1120 and 1145 (Tetrazole ring) ${ }^{1} \mathrm{H}$ NMR: $2.35(\mathrm{~d}, 6 \mathrm{H}$, $\left.\mathrm{CH}_{3}\right)$, 6.5-6.8 (d,1H,CH=CH) 7.30 -8.40 (m ,8H, Ar-H), $13.6(\mathrm{~s}, 1 \mathrm{H}, \mathrm{SH})$, Mass spectrum $(\mathrm{m} / \mathrm{z})$ molecular ion peak at 348 .

\subsubsection{4b: 5-[5-(2,6-dimethylphenyl)-1H-tetrazol-1-yl]-4-} (furan-2-yl)pyrimidin2-thiol

IR: $3050(\mathrm{Ar}-\mathrm{CH}), 1535(\mathrm{C}=\mathrm{N}), 1436(\mathrm{C}=\mathrm{C}), 1286(\mathrm{~N}-\mathrm{N}=\mathrm{N}-$ ),1120 and 1145 (Tetrazole ring), $780(\mathrm{C}-\mathrm{Cl}) .,{ }^{1} \mathrm{H}$ NMR: $2.35\left(\mathrm{~d}, 8 \mathrm{H}, \mathrm{CH}_{3}\right), 6.5-6.8(\mathrm{~d}, 1 \mathrm{H}, \mathrm{CH}=\mathrm{CH}) \quad 7.30 \quad-8.40$ $(\mathrm{m}, 8 \mathrm{H}$, Ar-H), $13.6(\mathrm{~s}, 1 \mathrm{H}, \mathrm{SH})$, Mass spectrum $(\mathrm{m} / \mathrm{z})$ molecular ion peak at 350 .

\subsubsection{4c:5-[5-(2,6-dimethylphenyl)-1H-tetrazol-1-yl]-4-}

(5-methylfuran-2-yl)pyrimidin-2-thiol

IR: $3052(\mathrm{Ar}-\mathrm{CH}), 1544(\mathrm{C}=\mathrm{N}), 1448(\mathrm{C}=\mathrm{C}), 1286(\mathrm{~N}-\mathrm{N}=\mathrm{N}-$ ),1245(-OCH3), 1120 and 1145(Tetrazole ring) , ${ }^{1} \mathrm{H}$ NMR: $2.35\left(\mathrm{~d}, 9 \mathrm{H}, \mathrm{CH}_{3}\right), 6.5-6.8(\mathrm{~d}, 1 \mathrm{H}, \mathrm{CH}=\mathrm{CH}) \quad 7.30 \quad-8.40$ $(\mathrm{m}, 7 \mathrm{H}$, Ar-H), $13.6(\mathrm{~s}, 1 \mathrm{H}, \mathrm{SH})$, Mass spectrum $(\mathrm{m} / \mathrm{z})$ molecular ion peak at 364 .

\subsubsection{4d:45-[5-(2,6-dimethylphenyl)-1H-tetrazol-1-yl]-} 4-(5-methylthiophen-2-yl)pyrimidin-2-thiol

IR: 3058 (Ar-CH), 1543(C=N), 1560(-NO2),1442(C=C), 1286(N-N=N-), 1120 and 1145 (Tetrazole ring), ${ }^{1} \mathrm{H}$ NMR: $2.35\left(\mathrm{~d}, 9 \mathrm{H}, \mathrm{CH}_{3}\right), 6.5-6.8(\mathrm{~d}, 1 \mathrm{H}, \mathrm{CH}=\mathrm{CH}) \quad 7.30 \quad-8.40$ $(\mathrm{m}, 7 \mathrm{H}$, Ar-H), $13.6(\mathrm{~s}, 1 \mathrm{H}, \mathrm{SH})$, Mass spectrum $(\mathrm{m} / \mathrm{z})$ molecular ion peak at 380 .

2.3.11. 4e:5-[5-(2,6-dimethylphenyl)-1H-tetrazol-1-yl]-4(3-methylthiophen-2-yl)pyrimidin-2-thiol

IR:3050 (Ar-CH), 1542(C=N), 1442(C=C), 1331($\mathrm{N}(\mathrm{CH} 3) 2,1286(\mathrm{~N}-\mathrm{N}=\mathrm{N}-), \quad 1120$ and 1145 (Tetrazole 
ring), ${ }^{1} \mathrm{H}$ NMR: $2.35\left(\mathrm{~d}, 9 \mathrm{H}, \mathrm{CH}_{3}\right), 6.5-6.8(\mathrm{~d}, 1 \mathrm{H}, \mathrm{CH}=\mathrm{CH})$ $7.30-8.40(\mathrm{~m}, 7 \mathrm{H}, \mathrm{Ar}-\mathrm{H}), 13.6(\mathrm{~s}, 1 \mathrm{H}, \mathrm{SH})$, Mass spectrum $(\mathrm{m} / \mathrm{z})$ molecular ion peak at 380 and isotopic peak at 381 .

\subsubsection{4f:5-[5-(2,6-dimethylphenyl)-1H-tetrazol-1-yl]-4-} (pyridin-2-yl)pyrimidin-2-thiol

IR: $\quad 3051(\mathrm{Ar}-\mathrm{CH}), \quad 1540(\mathrm{C}=\mathrm{N}), \quad 1442(\mathrm{C}=\mathrm{C})$, $1355(\mathrm{CH} 3), 1286(\mathrm{~N}-\mathrm{N}=\mathrm{N}-), 1120$ and $1145($ Tetrazole ring), ${ }^{1} \mathrm{H}$ NMR: $2.35\left(\mathrm{~d}, 9 \mathrm{H}, \mathrm{CH}_{3}\right), 6.5-6.8(\mathrm{~d}, 1 \mathrm{H}, \mathrm{CH}=\mathrm{CH})$ $7.30-8.40(\mathrm{~m}, 9 \mathrm{H}, \mathrm{Ar}-\mathrm{H}), 13.6(\mathrm{~s}, 1 \mathrm{H}, \mathrm{SH})$, Mass spectrum $(\mathrm{m} / \mathrm{z})$ molecular ion peak at 361 .

\section{Results and Discussion}

The reaction of 5-(2,6-dimethylphenyl)-1H-tetrazole with acetic anhydride to yield 1-[5-(2,6-dimethylphenyl)- $1 \mathrm{H}$ tetrazol-1-yl] ethanone (1) and which then treated with different aromatic aldehydes in presence of alkaline medium to form(2E)-1-[5-(2,6-dimethylphenyl)- $1 \mathrm{H}$ tetrazol-1-yl]-3-(substituted aryl) prop-2- en-1-one (2a-f). Reaction of (2a-f) with urea and thiourea to produce 5-[5(2,6-dimethylphenyl)-1H-tetrazol-1-yl]-4-(substituted aryl ) pyrimidin-2-ol (3a-f) and 5-[5-(2,6-dimethylphenyl)-1Htetrazol-1-yl]-4-(substituted aryl )pyrimidin-2-thiol (4a-f) respectively. The FT-IR spectra of (3a-f) and (4a-f) showed the absence of peak of keto groups and the new peaks which appeared at $3510 \mathrm{~cm}^{-1}$ due to $\mathrm{OH}$ group. This signifies the keto enol tautomeric in synthesized compounds. Some spectral data are listed in Table 2. The FT-IR absorption bands disappearance at $(1610-1650) \mathrm{cm}^{-1}$ is give good evidence for the success step of reaction. These absorption bands due to $(\mathrm{NH})$ amine group stretching frequency is at $3450 \mathrm{~cm}^{-1}$. Also FT-IR spectra of tetrazole showed clear absorption bands at $1286(\mathrm{~N}-\mathrm{N}=\mathrm{N}-), 1120$ and 1145 (Tetrazole ring). The NMR spectra of all the synthesized compounds were determined and the chemical shift values of $\mathrm{H}^{1} \mathrm{NMR}$ shows at $2.35\left(\mathrm{~d}, 9 \mathrm{H}, \mathrm{CH}_{3}\right), 6.5-6.8(\mathrm{~d}, 1 \mathrm{H}$, $\mathrm{CH}=\mathrm{CH}) 7.30-8.40(\mathrm{~m}, 9 \mathrm{H}, \mathrm{Ar}-\mathrm{H}), 9.5(1 \mathrm{H}, \mathrm{Ar}-\mathrm{OH})$, $13.6(\mathrm{~s}, 1 \mathrm{H}, \mathrm{SH})$. This values indicates the presence of all protons which conforms the structure of the synthesized compounds.

\subsection{Antibacterial and antifungal activity}

The antibacterial activity was evaluated by zone of inhibition method at $100 \mu \mathrm{g} / 0.1 \mathrm{ml}$ concentration. The results of antibacterial were compared with standard drug ciprofloxacin. Most of the synthesized compounds showed antibacterial activity against the tested Bacteria. It is evident that most of the compounds are very weakly active and few are moderately active against $S$. aureus and $E$. coli but compounds $3 \mathrm{c}, 3 \mathrm{~d}$ and $3 \mathrm{f}$ and compounds $4 \mathrm{c}, 4 \mathrm{~d}$ and $4 \mathrm{f}$ possess very good activity against $S$. aureus and $E$. coli at concentration of $100 \mathrm{ug} / 0.1 \mathrm{ml}$.

Similarly, the results of preliminary antifungal bioassays were compared with standard drug clotrimazole. Most of the synthesized compounds showed antifungal activity against the tested fungi. It is evident that the compounds $3 \mathrm{e}, 3 \mathrm{c}$ and $3 \mathrm{a}$ and compounds $4 \mathrm{e}, 4 \mathrm{~b}$ and $4 \mathrm{c}$ possess very good activity against fungi Candida albicans and Aspergillus niger at concentration of $100 \mathrm{ug} / 0.1 \mathrm{~mL}$.Compound $3 \mathrm{~d}$ and $4 \mathrm{f}$ showed moderate activity all bacteria and fungi tested

\section{Conclusion}

Tetrazole and Pyrimidine an important group of heterocyclic compounds reported to have different biological activities and hence the present studies were undertaken in order to synthesize Tetrazole clubbed with pyrimidines in order to potentiate the combined therapeutic effect of both heterocyclic compounds. So all the synthesized compounds were investigated them for their antibacterial and antifungal activity. Compounds with thiopene, furan and pyrrole substituents on pyrimidine clubbed with Tetrazole exhibited significant antibacterial and antifungal activity when compared with control. The compounds with pyrimidines substituents groups showed significant activity when compared to standard drug ciprofloxacin and clotrimazole respectively.

\section{Source of Funding}

None.

\section{Conflict of Interest}

None.

\section{References}

1. Stuart AL, Ayisi NK, Tourigny G, Gupta VS. Antiviral activity, antimetabolic activity, and cytotoxicity of $3^{\prime}$-substituted deoxypyrimidine nucleosides. J Pharm Sci. 1985;74(3):246-9. do1:10.1002/jps.2600740305

2. Kumar S, Narasimhan B. Therapeutic potential of heterocyclic pyrimidine scaffolds. Chem Cent J. 2018;12(1):1-29. doi: $10.186 / \mathrm{s}[3065-(0) 8-(0406-5]$

3. Suryawanshi SN, Kumar S, Shivahare R, Pandey S, Tiwari A, Gupta S. Design, synthesis and biological evaluation of aryl pyrimidine derivatives as potential leishmanicidal agents. Bioorganic Med Chem Lett. 2013;23(18):5235-8. do1:10.1016/].bmcl.2013.06.060.

4. Ahmed NM, Nofal S, Awad SM. Synthesis, Molecular Modelling and Biological Evaluation of Novel Pyrimidine Derivatives as Anti-inflammatory Agents. J Pharm Res Int. 2020;32(22):49-67. d01:10.9734/jpr1/2020/v3212230771.

5. Sherbiny F. Synthesis and Biological Evaluation of Novel Pyrazolo[3,4-D]Pyrimidine Derivatives of Expected Anticancer Activity. Al-Azhar J Pharm Sci. 2021;63(1):86-100. doi:1021608/AJPS.2021. 53563 .

6. Agarwal A, Chauhan S, Ashutosh N, Goyal PMS. Design, synthesis and biological evaluation of aryl pyrimidine derivatives as potential leishmanicidal agents. Bioorganic Med Chem. 2005;13(24):6678-84.

7. Sharma V, Chitranshi N, Agarwal AK. Significance and Biological Importance of Pyrimidine in the Microbial World. Int J Med Chem. 2014;2014:1-31. ब01:1//155/2014/202/84

8. Tauchi T, Ohyashiki K. The second generation of BCR-ABL tyrosine kinase inhibitors. Int J Hematol. 2006;83(4):294-300. 10i: $10.1532 / 2 \mathrm{H} 97.06025$. 
9. Selvam T, James C, Vijaysarathy P, Dniandev SK. A mini review of pyrimidine and fused pyrimidine marketed drugs. Res Pharm. 2012;2(4):1-9.

10. Solankee A, Kapadia K, Ćirić A, Soković M, Doytchinova I, Geronikaki A. Synthesis of some new S-triazine based chalcones and their derivatives as potent antimicrobial agents. Eur J Med Chem. 2010;45(2):510-8.

11. Sondhi SM, Dinodia M, Rani R, Shukla R, Raghubir R. Synthesis, anti-inflammatory and analgesic activity evaluation of some pyrimidine derivatives. Indian $J$ Chem Sec B Org Med Chem. 2009;48(2):273-81. doi:10.1016/j.bmc.2005.06.063.

12. Frère JM. Beta-lactamases and bacterial resistance to antibiotics. Mol Microbiol. 1995;16(3):385-95.

13. Mohamed NR, Saidi MMT, Ali YM, Elnagdi MH. Utility of 6amino-2-thiouracil as a precursor for the synthesis of pyrido[2,3d]pyrimidines and their in vitro and in vivo biological evaluation. Sci Pharm. 2007;75(1):9-28. doi:10.1016/j.bmc.2007.06.023.

14. Mostafa YAH, Hussein MA, Radwan AA, Kfafy AEHN. Synthesis and antimicrobial activity of certain new 1,2,4-triazolo[1,5-a] pyrimidine derivatives. Arch Pharm Res. 2008;31(3):279-93.

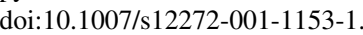

15. Bhalgat CM, Ali M, Ramesh B, Ramu G. Novel pyrimidine and its triazole fused derivatives: Synthesis and investigation of antioxidant and anti-inflammatory activity," Arab. J Chem. 2014;7(6):986-93.

16. Mohamed MM, Khalil AK, Abbass EM, El-Naggar AM. Design, synthesis of new pyrimidine derivatives as anticancer and antimicrobial agents. Synth Commun. 2017;47(16):1441-57.

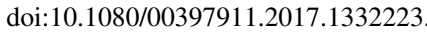

17. Farghaly AM, Aboulwafa OM, Elshaier YAM, Badawi WA, Haridy $\mathrm{HH}$, Mubarak HAE. Design, synthesis, and antihypertensive activity of new pyrimidine derivatives endowing new pharmacophores. Med Chem Res. 2019;28(3):360-79.

18. Alam O, Mullick P, Verma SP, Gilani SJ. Synthesis, anticonvulsant and toxicity screening of newer pyrimidine semicarbazone derivatives. Eur J Med Chem. 2010;45(6):246772. [01:10.1016/1.ejmech.2010.02.031.

19. Radwan MAA, Alshubramy MA, Motaal MA, Hemdan BA, ElKady DS. Synthesis, molecular docking and antimicrobial activity of new fused pyrimidine and pyridine derivatives. Bioorg Chem. 2019;96:103516. doi:10.1016/1.bioorg.2019.103516.
20. Abbass AF, Zimam EH. Synthesis, characterization and study biological activity of some new pyrimidine and 1, 2, 3, 4tetrazole derivatives based on sulfadiazine. Int J ChemTech Res. 2016;9(11):206-17.

21. Mohite P, Pandhare RB, Khanage SG, Bhaskar VH. Synthesis and Anti-Inflammatory Activity of Some 5-Phenyl-1-(Acyl)-1, 2, 3, 4Tetrazole. J Pharm Res. 2010;3(1):43-6.

22. Leal JG. Synthesis and electrochemical and antioxidant properties of chalcogenocyanate oxadiazole and 5-heteroarylchalcogenomethyl1: H -tetrazole derivatives. New J Chem. 2017;41(13):5875-83.

23. Bhaskar VH, Mohite PB. Synthesis, characterization and evaluation of anticancer activity of some tetrazole derivatives. J Optoelectron Biomed Mater. 2010;2(4):249-59.

24. Vembu S, Pavadai P, Gopalakrishnan M. Synthesis, in vitro antifungal and antitubercular evaluation of novel amino pyrimidines based tetrazole derivatives. J Pharm Res. 2014;8(10):1552-8.

25. Vellalacheruvu R, Leela S. Novel Route for Synthesis of Antihypertensive Activity of Tetrazole Analogues as a Carbamate and Urea Derivatives. Med Chem . 2017;07(08):1-8. 10i:10.4172/216] 0444.000463.

26. Liu DC, Zhang HJ, Jin CM, Quan ZS. Synthesis and biological evaluation of novel benzothiazole derivatives as potential anticonvulsant agents. Molecules. 2016;21(3):164. Hoi: 103300/molecules21030164.

\section{Author biography}

N.D. Bhoge, Assistant Professor

B.K. Magare, Professor

P. B. Mohite, Professor

Cite this article: Bhoge ND, Magare BK, Mohite PB. Synthesis, characterization and antimicrobial activity of novel tetrazoles clubbed with pyrimidine. J Pharm Biol Sci 2021;9(2):116-122. 